\title{
USING WAVELETS IN ECONOMICS. AN APPLICATION ON THE ANALYSIS OF WAGE-PRICE RELATION
}

\author{
Vasile-Aurel Caus ${ }^{1}$, Daniel Badulescu ${ }^{2 *}$, Mircea Cristian Gherman ${ }^{3}$ \\ ${ }^{1}$ Department of Mathematics, University of Oradea, Oradea, Romania \\ ${ }^{2}$ Department of Economics, University of Oradea, Oradea, Romania \\ ${ }^{3}$ Technical University of Cluj-Napoca, Romania \\ vcaus@uoradea.ro \\ dbadulescu@uoradea.ro \\ mircea.gherman@com.utcluj.ro
}

\begin{abstract}
In the last decades, more and more approaches of economic issues have used mathematical tools, and among the most recent ones, spectral and wavelet methods are to be distinguished. If in the case of spectral analysis the approaches and results are sufficiently clear, while the use of wavelet decomposition, especially in the analysis of time series, is not fully valorized. The purpose of this paper is to emphasize how these methods are useful for time series analysis. After theoretical considerations on Fourier transforms versus wavelet transforms, we have presented the methodology we have used and the results obtained by using wavelets in the analysis of wage-price relation, based on some empirical data. The data we have used is concerning the Romanian economy - the inflation and the average nominal wage denominated in US dollars, between January 1991 and May 2016, highlighting that the relation between nominal salary and prices can be revealed more accurately by use of wavelets.
\end{abstract}

Key-words: wage-price spiral, wavelet transforms, Fourier transforms, spectral analysis.

JEL classification: C02, C32, E31.

\section{Introduction}

The last decades witnessed the development of mathematical tools dedicated to economic analysis. Starting with Nash's pioneering work, there is an increased concern for searching economic applications of mathematical methods and tools. In the same time, the problem of evaluating and testing the utility of used mathematical methods has emerged. Analysis of economic phenomena is, by its nature, intended to identify components and their impact on the fulfillment of economic processes. Most often this operation is difficult and involves the use of mathematically complex tools, among which wavelet method is to be mention.

The objective of this article is to emphasize the usefulness of these methods in time series analysis. The article is organized as follows. In the first part we present how Fourier and wavelet transforms are used in economics, and the differences between the two methods. In the second part we present the methodology used, in the third part we present the findings and results obtained by using wavelet analysis applied on wage-price relation, based on some empirical data, and finally we conclude.

\footnotetext{
* Corresponding author
} 


\section{Theoretical overview on wavelet transforms used in economics}

The wavelets theory has developed very rapidly in the last twenty years. There are various applications of wavelets, related to: signal analysis, astronomy, medicine (especially imagistic), geology, meteorology, physics, etc. We would have expect that dual time-frequency properties and the ability to work both on stationary and non-stationary series recommend wavelets as an effective mathematical tool in economics, especially in financial macroeconomics. However, there are few works dealing with the application of wavelets in economics. Therefore, we consider that any contribution highlighting the efficiency and consistency of the "wavelet analysis" in economics is useful in developing economic modeling techniques.

Time series analysis is probably the most important chapter of econometrics (and not only). The main purpose (in economic terms) of this type of analysis is to identify a function or at least an argument to allow a forecast of the future values of the series in question. In other words, in most cases, it is about predicting the future based on past analysis. Economic phenomena represented by time series and subject of analysis are not always simple; on the contrary, they are often difficult to analyze. This is due, in most cases, to the difficulty of identifying and isolating the analyzed components of the phenomenon (trend, seasonality, etc.). Therefore, the analysis of the economic phenomena, time related, although efficient in many situations, is insufficient in other cases. Graphs, so common in economic presentations, are mostly in the time-amplitude area: on the horizontal axis time is represented, and on the vertical axis the values - measured, calculated or predicted - of the time-dependent variable. This representation, while very often useful, hides, in the case of complex phenomena, the most important features. This is the main reason for developing mathematical tools to transform these representations into more suggestive ones.

The stated purpose of applying transforms on economic time series is to identify the main components of the phenomena, the frequency and the amplitude of them. Once identified and isolated these components, they can be easier analyzed and their behavior can be predicted with a higher degree of accuracy than in the case of the time-analysis of the original series. Since the original series can be reconstructed based on the components, the prediction arguments developed on the latter ones can be extended to the original series, considering, of course, the interdependence between them.

A first transform used in many economic analyses is the Fourier transform (continuous, discrete, fast etc.). Basically, by applying this transformation, we pass from the time-domain into the frequency-domain. In other words, the horizontal axis shows frequencies, and the vertical axis shows amplitudes. A crucial observation is that, when using Fourier transform, the graph does not contain time. Specifically, Fourier transform can tell us which are the frequencies of the main components of the analyzed phenomenon, but it cannot provide information about the moments, in time, when these frequencies occur. Even if, as we mentioned, the original series can be reconstructed from Fourier transform, in other words we can pass anytime from one representation to another (from the time domain to frequency domain and vice versa) we cannot obtain simultaneously information on time, frequency, amplitude. For this reason Fourier transformation is used only for stationary series (series which do not change their frequency structure over time).

There is a rich literature on Fourier analysis (or spectral analysis). The first important treaty is C.W.J. Granger's "Spectral analysis of economic time series" (1964). Another master work is M.B. Priestley's "Spectral analysis and time series" (1981), but also dedicated book chapters in Hamilton's "Time Series Analysis" (1994) or Handbook of Econometrics, Volume II (Griliches and Intriligator, editors) (1984). Among the recent works, we mention Bloomfield's "Fourier Analysis of Time Series" (2000) and Broersen's "Automatic Autocorrelation and Spectral Analysis" (2006). Of course, there are hundreds of articles published in academic journals. 
Unfortunately, as we mentioned, the Fourier transform is inefficient for non-stationary series because it provides single information: the spectral composition or spectrum (which frequencies and amplitudes do the components of the analyzed series have), without being able to highlight the changes during time of the spectrum. As expected, the spectral analysis relies on the term "light spectrum". Just as light, it can be split into several components with different frequency composing the spectrum and which are invisible to the eye unless a transformation is used (e.g. a prism in the beam path), so that a phenomenon represented by a time series may contain components undetectable in the absence of transformation.

The Fourier transform default is related to the failure to provide information on the time location of the spectral structure. If an economic series needs a time-frequency representation it is necessary to use another transform. Before presenting this more powerful transformation, we have to remember that the use of Fourier transform with some restrictions generate variants (short time Fourier transform, windowed Fourier transform) that allow a time-frequency analysis. This can be done, for example, by using frequency filters which decompose the signal in frequency bands with given width. The 3D representation of this decomposition uses one time axis, one for frequencies and one for amplitude. This representation is most of the time unsatisfactory because doesn't allow to modify the resolution (more information about this scale is found in the works mentioned in the bibliography, especially those relating to wavelets).

This brings us, finally, to wavelets. Wavelets, as suggested by their names, are small waves. The most important feature is that they have local support, in other words, their value is different than zero on a finite and bounded interval, but in the rest they have the zero value. Why is this important? Let us return to Fourier transform. Although we haven't presented the analytical expression of this transformation, we can imagine that changing a coefficient in the expression of Fourier transform attached to a function or to a discrete series produces changes spread through all the period (i.e. changes cannot be located in time). In other words, let's suppose that we intervene on a point of a metal band swing. Our intervention will affect the band swings from one end to another. Thus, we cannot analyze the effect of spectral changes at some given moment as these changes are transmitted through the whole series.

There is an essential difference between wavelet transform and Fourier transform. Because wavelets have non-zero values on sufficiently small intervals, when you change the coefficients in the series representation through wavelets, the effect diminishes quickly enough. Because, finally, everything comes down to this: the determination of the values or analytical expressions of some coefficients in order to obtain a better (or the best) approximation of the dynamics of an economic phenomenon.

As we mentioned, there is yet another important distinction between Fourier transform and wavelet transform. The latter allows a multi-resolution analysis, while Fourier analysis does not allow changing the resolution. The resolution adaptation (or change) on the spectrum series is very important. It is obvious that, at a low resolution, the image can be seen entirely, but details are lost; while at high resolution we identify details, but the overall picture is lost. Therefore, the multi-resolution analysis uses high resolution for time and small for frequency in the case of high frequencies, respectively small resolutions for time and high resolutions for frequencies in the case of low frequencies. The reason for which we cannot use simultaneously high resolutions for both time and frequencies is derived from Heisenberg's uncertainty principle. This is the reason determining, when necessary, the use of transformations (as it is the wavelet transformation) allowing the adaptation of the resolution. If we consider economic time series, in most cases they contain a low frequency and longtime duration trend and seasonality or disturbance of (relatively) high frequency with a short event.

The wavelet transformation consists mainly in the application of an operator on the regression function constructed on the base of previous observations. 
First, we note that this transformation uses two categories of wavelets: mother wavelets and father wavelets. The mother wavelets have the property that:

$$
\int \psi(t) d t=0,
$$

and father wavelets have the property that:

$$
\int \phi(t) d t=1
$$

Any function $f(t)$ can be represented as a linear combination of mother and father wavelets. If, for example, $\psi(t)$ and $\phi(t)$ are symmetrical mother and father wavelets (so called symmlets), then they can be expressed as:

$$
\psi_{j, k}(t)=2^{-j / 2} \psi\left(2^{-j} t-k\right), j, k \in Z
$$

and

$$
\phi_{j, k}(t)=2^{-j / 2} \phi\left(2^{-j} t-k\right), j, k \in Z
$$

Obviously, symmetrical wavelets are not the only wavelets that can be used in practical applications.

The $f(t)$ function can therefore be represented as:

$$
f(t)=\sum_{k} s_{N, k} \phi_{N, k}(t)+\sum_{k} d_{N, k} \psi_{N, k}(t)+\sum_{k} d_{N-1, k} \psi_{N-1, k}(t)+\ldots+\sum_{k} d_{1, k} \psi_{1, k}(t)
$$

where $s_{N, k} \approx \int f(t) \phi_{N, k}(t) d t$ and $d_{j, k} \approx \int f(t) \psi_{j, k}(t) d t, j \in\{1,2, \ldots, N\}$ are the transformation coefficients.

In this representation $j$ is the scale used, and $k$ is the number of translations corresponding to each scale, while $N$ is the maximum value of the scale used in the representation. The functions $\psi_{N, k}(t)$ and $\phi_{N, k}(t)$ must fulfill the condition that they form an orthogonal basis. If we denote $S_{N}=\sum_{k} s_{N, k} \phi_{N, k}(t)$ and $D_{N}=\sum_{k} d_{N, k} \psi_{N, k}(t)$, then multi-resolution decomposition of the function $f(t)$ is given by $\left\{S_{N}, D_{N}, D_{N-1}, \ldots, D_{1}\right\}$.

The described representation corresponds to a discrete variable (the most common case encountered in economic data analysis) and the transformation is called transformation by discrete wavelets, unlike the continuous case (wavelets continuous transformation) using integral operators.

The information on time series are obtained by the analysis of partial sums,

$$
S_{j-1}(t)=S_{N}(t)+D_{N}(t)+D_{N-1}(t)+\ldots+D_{j}(t),
$$

providing information on the dynamics (frequency and time) of the components that are most relevant for the series' behavior. Once these components are identified, they can be "cleaned" and assembled in order to build an approximation of the original series. 
A graphical scheme that explains easy the algorithm of using the discrete transformation by wavelets and the use of the inverse transform (for the series' reconstruction) can be found in Crowley (2007).

\section{Methodology: thresholding with wavelets}

Economic time series and financial data contain information which is sometimes considered to be un-useful or unwanted. For better prediction accuracy, it is necessary to smooth the data or to filter out the noise (perturbation). This technique is often called de-noising and can be accomplished with wavelets transforms too, see Donoho and Johnstone (1995). A de-noising algorithm could be used in order to "clean" any signal (time series) that contains the unwanted noise (Karim, et al., 2011). Since the wavelet transform is a set of coefficients representing the time series in a wavelet domain, the techniques applied in this domain will affect also the series in the time domain. One reason of performing some processing on other domain is that the detail and approximations scales are very well defined and also very clearly represented in such kind of transformations. As a consequence, wavelet filtering acts as a smoothing operator. Not the same can be affirmed about Fourier basis. By shrinking Fourier coefficients, we can get bad results in terms of mean square error. Also, some visual artifacts can be observed in the filtered time series as stated in Donoho (1993).

The filtering technique in wavelet domain is often called thresholding and it is based on the idea that the majority of wavelet coefficients correspond to details (Al Wadi, et al., 2011). When details are small, they might be omitted without substantially affecting the "general picture." Thus the idea of thresholding wavelet coefficients is a way of cleaning out "unimportant" details considered to be noise.

Thresholding is a good technique in time series processing due to the fact that the parsimony of wavelet transformations ensures that the signal of interest can be well described by a relatively small number of wavelet coefficients.

The process of thresholding wavelet coefficients can be divided into two steps. The first step is the choice of the threshold function $T$. There are two main rules of thresholding: soft thresholding or hard thresholding. Given a wavelet coefficient $w$ and threshold value $\lambda$, the corresponding transformation for hard threshold value of the coefficient can be written as (Karim, et al., 2011):

$$
T_{\text {hard }}(w, \lambda)=w I(|w|>\lambda),
$$

On the other hand, for the soft threshold the previous transformation becomes:

$$
T_{\text {soft }}(w, \lambda)=\operatorname{sign}(w)(|w|-\lambda) I(|w|>\lambda) \text {, Where } / \text { is the usual indicator function. }
$$

In other words, "hard" means "keep or kill" while "soft" means "shrink or kill", see Hardle et al. (1998) or Van Fleet (2008). The commonly adopted rule is to use soft thresholding. Although the hard thresholding is able to preserve the peak, it also produces more spurious oscillations and close discontinuities (Karim et al., 2011).

The second step is the choice of a threshold. In the following sections we briefly present some considerations about methods of selecting a threshold. In financial applications (Ramsey, 2002), soft thresholding methods or some combined method between the two are often used. Depending on the application, other types of thresholding can be used, which have several approaches. Wang (1994) generalizes Nason's cross validation technique by removing more than half of the data each time. The motivation is to improve the robustness of the threshold selection procedure against the effect of a correlated noise (with long range dependence). Saito (1994) incorporates the hard thresholding into a minimum description length procedure. Vidakovic (1994) describes wavelet shrinkage via Bayes rules and Bayesian testing of hypothesis. 
In this study we have applied the modified thresholding algorithm based on the Donoho and Johnstone (1995) threshold method. This is based on some prerequisite assumptions. If $z$ is a random variable having its elements $z_{i}$ independent and normal identically distributed (i.i.d. $-z_{i} \sim \boldsymbol{N}(\mathbf{0 , 1})$ ), then it can be defined the following variable:

$$
A_{n}=\left\{\max \left|z_{i}\right| \leq \sqrt{2 \log n}\right\} ; i=1, n \text {. }
$$

The $\boldsymbol{A}_{n}$ is defined in such a way that $\pi_{n}=\boldsymbol{P}\left(\boldsymbol{A}_{\boldsymbol{n}}\right) \rightarrow \mathbf{0 , n} \rightarrow \infty$. In addition, if $\left.\boldsymbol{B}_{n}(t)=\left\{\max \left|z_{i}\right|>t+\sqrt{2 \log n}\right\}\right\}$ for every sample of the $z$ dataset $(\mathrm{i}=1, \mathrm{n})$, then $P\left(B_{n}(t)\right)<e^{-t^{2} / 2}$.

That motivates the following threshold: $\lambda^{U}=\sqrt{2 \log n} \hat{\sigma}$, which is meant to be universal. In this expression the variance of the analyzed data is considered to be $\hat{\sigma}$.This threshold is one of the first proposed and provides a fast and, in the same time, easy and automatic thresholding. The reason behind this is to remove all wavelet coefficients that are smaller than the expected maximum of an assumed i.i.d. noise sequence of given size $(n)$. Hence, there are several possibilities to estimate the variance $\hat{\sigma}$ from the above expression.

Almost all thresholding methods involve the wavelet coefficients of the finest scale which are considered to be strongly related to the noise since these scales represent the most detailed elements of the analyzed time series. The signal-to-noise ratio is smallest at high resolutions in wavelet decomposition for almost all signals.

Some standard estimators are:

or a more robust

$$
\hat{\sigma}^{2}=\frac{1}{N / 2-1} \sum_{i=1}^{N / 2}\left(w_{n-1, i}-w\right)^{2}
$$

$$
\hat{\sigma}^{2}=1 / 0.6745 \cdot M A D\left(\left\{w_{n-1, i}, i=1, N / 2\right\}\right)
$$

Where $n$ is the highest level of the wavelet transform.

In some applications, especially if using the first estimator and the data sets are large, and when $\sigma$ is overestimated like in the case when using the second estimator, the universal thresholding can give under-fitted models.

As a matter of fact one can use a simple algorithm in order to de-noise a financial time series. The step of this kind of algorithm can be resumed as following:

1) First, based on its properties, choose the basis wavelet function and the number of scale levels for signal decomposition

2) Apply a wavelet transform to the original signal (time series) and create the matrix transformation

3) Remove the small (detail/wavelets) coefficients by using a thresholding method

4) Perform other processing or adjustments to the wavelet coefficients, if needed

5) Reconstruct the original signal by applying the inverse wavelet transform to the processed transformation.

After applying all these steps we obtain the reconstruct time series having some "new" properties based on which one can take different decisions in the economic field, including here the relation between inflation and nominal wages. 


\section{An application on wage-price relation. Findings and results}

In this section we consider an application concerning the relation between salaries (wages) and inflation (prices). We will briefly present a short theoretical overview on the relation investigated, and then present our findings related to the applied method regarding filtering and thresholding the economic time series.

During the last decades, finding a relation between wage growth and periodic inflation kept the attention of economists, hoping to understand the developments on the labor market which may be a good predictor of inflationary pressures. A clear argument for how labor costs tend to push up prices, i.e. the so-called wage-price spiral, is synthesized in Layard, Nickell and Jackman (1994). They stated that "when buoyant demand reduces unemployment (at least relative to recent experienced levels), inflationary pressure develops. Therefore, the companies start bidding against each other for labor, and workers feel more confident in pressing wage claims. If the inflationary pressure is too great, inflation starts spiraling upwards: higher wages lead to higher price rises, leading to still higher wage rises, and so on" (Layard et al., 1994). This is the wage-price spiral presented in the mentioned work. In respect to this, we can notice a re-evaluation of the cost push type models of inflation, the estimations and statistical analysis trying to see if the production cost channel is a credible mechanism for price increases. Another important approach is mentioned in Aristide (2007). Thus, if the aggregate demand is maintained constant, then the productivity, the competitive pressures and the profit rate of enterprises will increase the labor costs (also with fiscal taxes and raw materials) which will be reflected in increased economic prices (Badulescu and Caus, 2011).

Tracking statistics data indicates, at least apparently, an "in tandem" movement of wages and prices, but this cannot convince the researchers to unanimously consider that wages growth leads to rising prices, i.e. inflation. In fact, the last decades show that there are two main approaches analyzing and interpreting statistical data in wage-inflation relation.

On one hand, works such as Gordon (1988), Rissman (1995) quoted in Hess and Schweitzer (2000), Clark (1998), and, partially, Jonsson and Palmqvist (2004) state that there is no strong evidence of the relation between these two variables. On the other hand, we can mention the works of Ghali (1999) or Aaronson (2001) sustaining a significant relation between wage growth and inflation.

In the same trend, some papers and works undertaken by the European Central Bank show that, on the short run, an inflationary process can result from increases in factors such as consumption, investment, widening budget deficits, energy costs or wages uncorrelated with productivity gains. Inflationary pressure may also occur if the "workers and unions would have doubts about future inflation, and therefore they would require a considerable increase of nominal wages to avoid a strong future inflation leading to significant decreases in real wages" (European Central Bank, 2011).

As a conclusion to these approaches, we can say that increasing the unit labor cost may be an important element for a series of economic variables (such as the trade deficit), but it affects in lower measure the prices adjusted with productivity (Badulescu and Caus, 2010). We will try to identify whether there is or there is not a strong relation between wages and prices (inflation).

The data we have used is concerning the Romanian economy and it has been provided by the Romanian Institute of Statistics. The two economic series, the inflation and the average nominal wage denominated in US dollars are considered on a monthly basis. The covered period is from January 1991 to May 2016. 


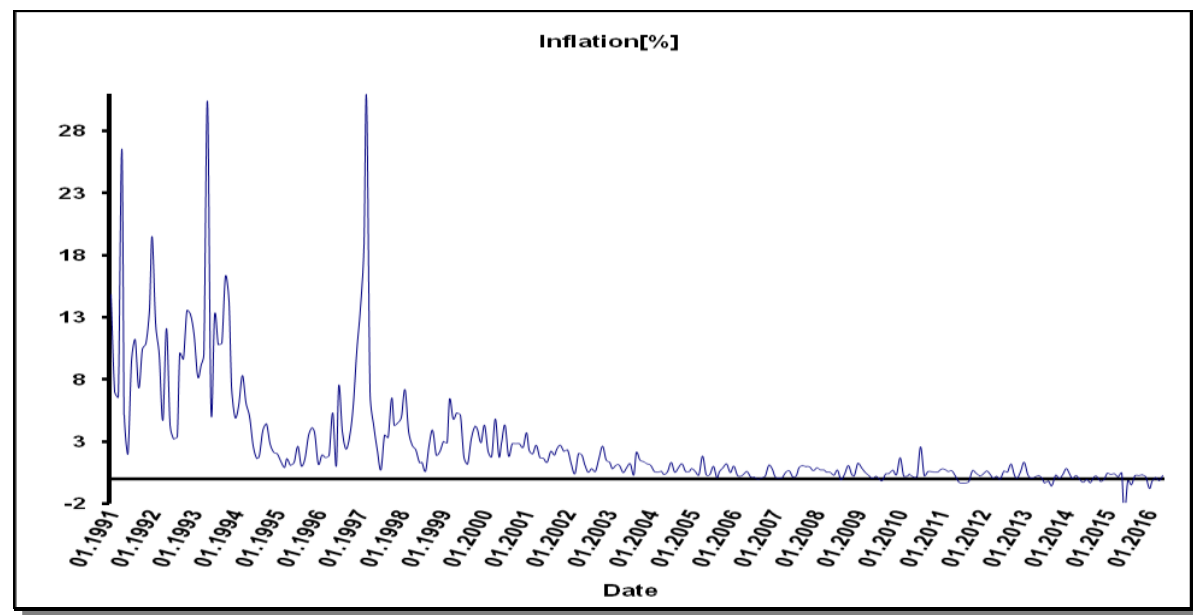

Figure 1: Evolution of inflation rate in Romania (1991-2016), in \%

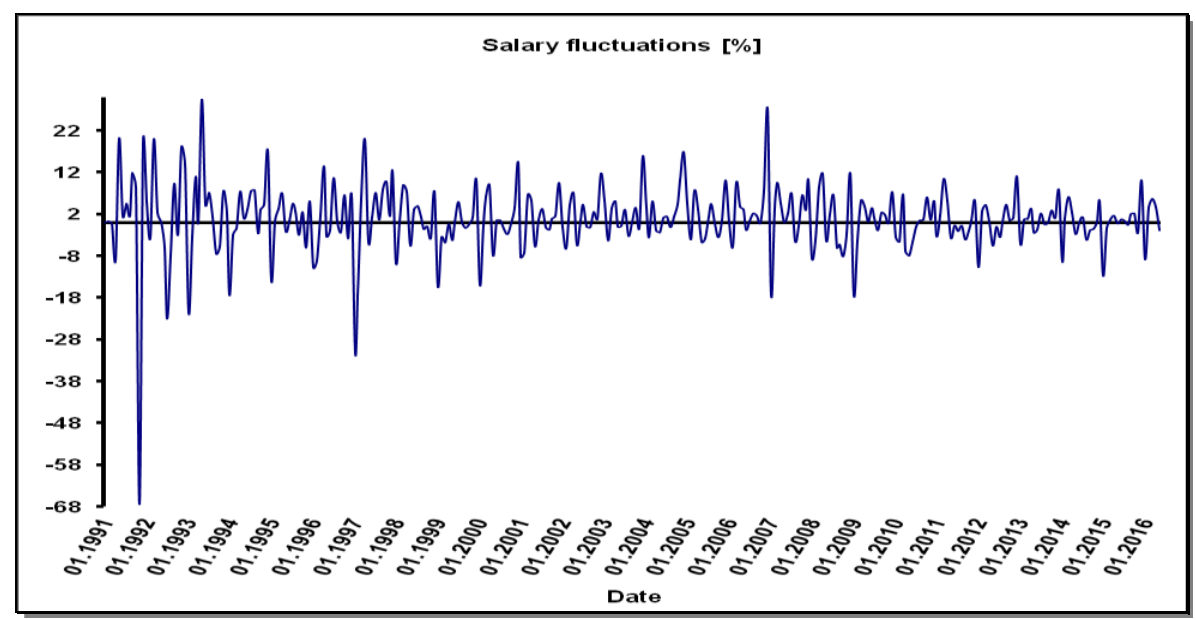

Figure 2: Salary fluctuation in Romania (1991-2016), in \%

Both time series are decomposed over an orthogonal wavelet basis. In this paper we chose the Symlet8 (sym8 wavelet) using the multi-level decomposing wavelet transform. The level decomposition, which is equal to the number of decomposition scales, has been chosen to be equal to 2 . Too many scales do not necessary increase the performance of wavelet filtering. Then we selected a part of the coefficients through thresholding and we keep the coefficients of approximation above the threshold value. The filtering is performed using a combination between hard and soft thresholding (Karim et al., 2011). A recent method has been used, which is implemented using an automatic switching mechanism between the classical thresholding methods. The filtering removes the noisy components from the original time series while preserving any spike or anomaly that exists in the beginning of processing. 


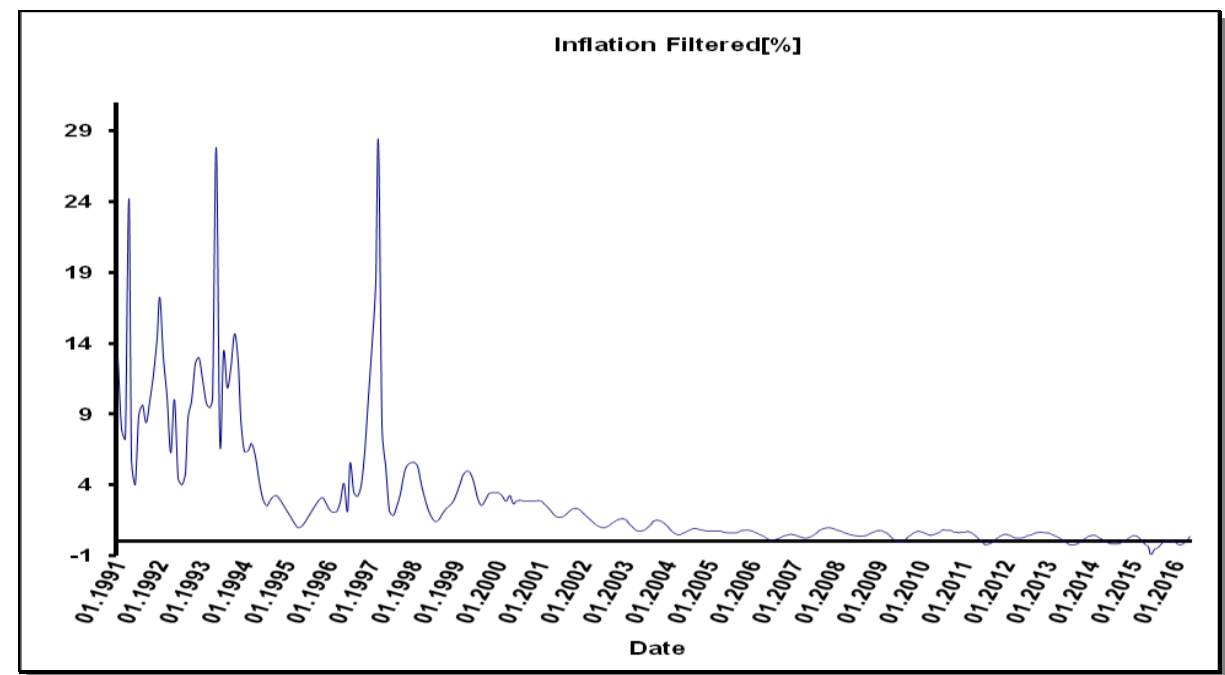

Figure 3: Filtered inflation rate in Romania (1991-2016), in \%

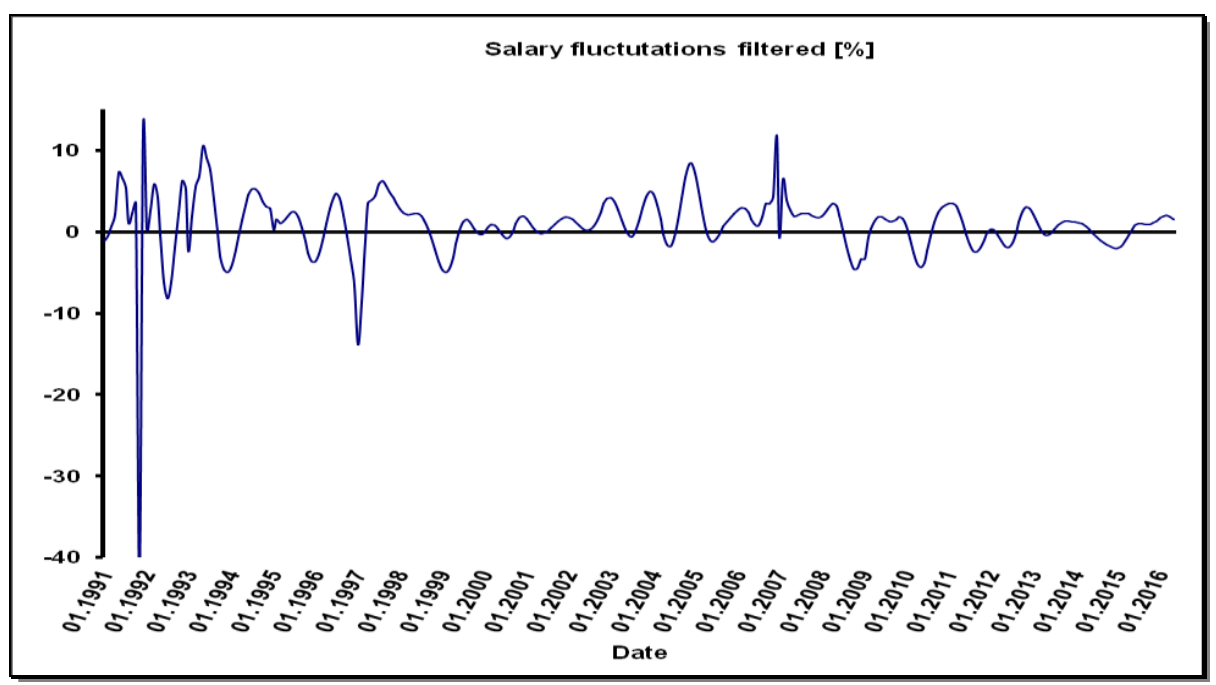

Figure 4: Filtered salary fluctuation in Romania (1991-2016), in \%

\section{In conclusion}

We can conclude that, by using the filtering technique using the wavelet transform, the relation between nominal salary and prices (inflation) can be revealed at a more accurate level than by using unfiltered data. The wavelet transform performs well in the period with lower volatility and smaller fluctuations. These periods of time were associated with the last 10-15 years when the economy became more stable and the inflation target policy of government and monetary authorities gave better results. At the beginning of the analyzed period, due to the fact that the economic instability was higher, wavelet de-noising couldn't achieve a very good performance from the accuracy of filtered data point of view. Thus, the wavelet transforms remain very powerful methods for data de-noising, and recommended for further analysis in the economic area. 


\section{References}

Aaronson, D. (2001). 'Price Pass-Through and the Minimum Wage'. Review of Economics and Statistics, vol. 83, no. 1, pp. 158-169.

Al Wadi, S., Mohd Tahir Ismail, Alkhahazaleh, M. H., Samsul Ariffin Abdul Karim (2011). 'Selecting Wavelet Transforms Model in Forecasting Financial Time Series Data Based on ARIMA Model'. Applied Mathematical Sciences, vol. 5, no. 7, pp. 315 - 326.

Aristide, O. (2007). 'Impactul salariilor din economie asupra inflatiei si deficitului commercial/ The impact of salaries on inflation and commercial deficit'. Caiete de Studii Banca Nationala a Romaniei/ Working Papers Romanian National Bank, 24, December, pp. 1-31.

Badulescu, A., Caus, V. (2010). The influence of the utility function on the dynamics of the underground economy. lasi, Conference Proceedings 11th WSEAS International Conference on Mathematics \& Computers in Business \& Economics.

Badulescu, A., Caus, V. (2011). 'The Underground Economy: What is the Influence of the Discount Factor?' African Journal of Business Management, vol. 5, no. 16, pp. 7050-7054. Bloomfield, P. (2000). Fourier analysis of time series: an introduction. New York: Wiley.

Broersen, P. (2006). Automatic Autocorrelation and Spectral Analysis. London: Springer-Verlag.

Clark, T. (1998). 'Employment Fluctuations in U.S. Regions and Industries: The Roles of National, Region-Specific, and Industry-Specific Shocks'. Journal of Labor Economics, University of Chicago Press, vol. 16, no. 1, pp. 202-229.

Crowley, P. (2007). 'A Guide To Wavelets For Economists'. Journal of Economic Surveys, vol. 21 , no. 2.

Donoho, D. (1993). Nonlinear Wavelet Methods for Recovery of Signals, Densities, and Spectra from Indirect and Noisy Data. Proceedings of Symposia in Applied Mathematics, American Mathematical Society.

Donoho, D., Johnstone, I. (1995). 'Adapting to Unknown Smoothness via Wavelet Shrinkage'. Journal of the American Statistical Association, vol. 90, no. 432, pp. 1200-1224. European Central Bank (2011). Price stability: Why is it important for you?. Available from: https://www.ecb.europa.eu/pub/pdf/other/price stability web 2011en.pdf. [10 November 2016].

Ghali, K. H. (1999). 'Government size and economic growth: Evidence from a multivariate cointegration analysis'. Applied Economics, vol. 31, pp. 975-987.

Gordon, R. (1988). 'The Role of Wages in the Inflation Process. The American Economic Review'. Papers and Proceedings of the One- Hundredth Annual Meeting of the American Economic Association, vol. 78, no. 2, pp. 276-283.

Granger, C., Hatanaka, M. (1964). Spectral analysis of economic time series. Princeton, NJ: Princeton.

Griliches Z., Intriligator M. (eds). (1984). Handbook of Econometrics, vol 2, Elsevier.

Hamilton, J. (1994). Time Series Analysis. Princeton, NJ: Princeton University Press.

Hardle, W., Kerkyacharian, G., Picard, D., Tsybakov, A. (1998). Wavelets, Approximation and Statistical Applications. Lecture Notes in Statistics, vol. 129, New York: Springer.

Hess, G., Schweitzer, M. (2000). 'Does Wage Inflation Cause Price Inflation?' Federal Reserve Bank Of Cleveland, Policy Discussion, 10.

Jonsson, M., Palmqvist, S. (2004). 'Do Higher Wages Cause Inflation?' Sveriges Riksbank Working Paper Series, Issue 159, pp. 1-48.

Karim, S.A.A., Karim, B.A., Ismail, M.T., Hasan, M.K., Sulaiman, J., (2011). 'Application of Wavelet Method in Stock Exchange Problem'. Journal of Applied Sciences, vol 11, pp. 1331-1335.

Layard, R., Nickell, S., Jackman, R. (1994). The Unemployment Crisis. Oxford: Oxford University Press.

Priestley, M. (1981). Spectral Analysis and Time Series. (Vol. 1\&2). London: Academic Press. 
Ramsey, J. (2002). 'Wavelets in economics and finance: Past and future'. Studies in Nonlinear Dynamics \& Econometrics, vol. 6, pp. 1-27.

Rissman, E. (1995). 'Sectoral Wage Growth and Inflation'. Federal Reserve Bank of Chicago.Economic Review, Volume July/August, pp. 16-28.

Saito, N. (1994). Simultaneous noise suppression and signal compression using a library of orthonormal bases and the minimum description length criterion. In: E. Foufoula-Georgiou \& P. Kumar, eds. Wavelets in Geophysics. New York: Academic Press, pp. 299-324.

Van Fleet, P. (2008). Discrete Wavelet Transformation: An Elementary Approach with Application. New Jersey: John Wiley \& Sons.

Vidakovic, B.(1994). Nonlinear wavelet shrinkage via Bayes rules and Bayes factor, Discussion Paper 94-24. ISDS, Duke University.

Wang, Y. (1994). Function estimation via wavelets for data with long-range dependence. Tech. Report, University of Missouri-Columbia: Department of Statistics.

\section{Bio-note}

Vasile-Aurel Caus is associate professor at the Department of Mathematics and Informatics, Faculty of Sciences, University of Oradea, and member of several professional organizations (AMS, SIAM, RGMIA, MAA, SPSR, ROMAI, SSMR). His research activity is focused on applied mathematics, particularly in economics.

Daniel Badulescu graduated Bucharest University of Economic Studies and currently is Professor and Ph.D. supervisor at the Doctoral School in Economics within the University of Oradea, Romania. He holds the position of director of the Research Centre for Competitiveness and Sustainable Development.

Mircea Gherman is researcher working with Technical University of Cluj-Napoca and consultant in information technology center for pharmaceutical and stock industries. As researcher, his activities are focused on continuing the development of mathematical models based on spectral and statistical analysis that were started during his $\mathrm{PhD}$ researches. He also maintains contact with stakeholders from innovation, analysis, IT and marketing areas of the companies for which he provides advices and technological solutions. 